\title{
Research and Application of High Temperature Resistant Water Shutoff Profile Control Agents
}

\author{
Meng Zheng \\ Drilling and Producing Technology Research Institute, Liaohe Oilfield, Panjin, 124010,China \\ Email: zhengmeng@petrochina.com.cn
}

Keywords: High temperature water shutoff profile control; Adjust the steam absorption profile; Improve the effect of steam injection

\begin{abstract}
After many rounds stimulation, high permeable zone of Liaohe oilfield heavy oil block has reached high recovery degree. Every steam injection didn't enter into the middle and low permeable formation, medium and low permeability layer recovery degree is still very low or unused, leading to low steam injection efficiency. It is necessary to adjust the steam absorption profile and improve the effect of steam injection to improve reservoir producing extent.In this paper, a series of high temperature resistant water shutoff profile control agents are developed in view of the heavy oil stimulation exploit using composite plugging technology to adjust steam absorption profile.
\end{abstract}

\section{Introduction}

In the process of heavy oil steam stimulation, Heterogeneity between layers and steam overlap phenomenon exists, the steam absorption differences will occur and high permeable formation is strong vapor absorption layer, low permeable formation is weak steam absorption layer, even don't absorb steam[1]. After several rounds of steam injection, steam channeling channel is produced, causing steam channeling interference between wells, which substantially reduce the steam stimulation effects.Currently one of the most effective way to solve this contradiction is high temperature chemical profile control, namely high temperature profile control agent technology. High temperature profile control agent must have a long term resistance characteristics of the steam injection temperature (above $300^{\circ} \mathrm{C}$ ), and has a certain resistance to steam erosion intensity, which ensure that the steam is not blocked into the horizon after implementation[2].

A series of high temperature resistant water shutoff profile control agent was developed for the heavy oil stimulation using high, medium temperature plugging agent to adjust steam absorption profile.

\section{Research and evaluation of high temperature resistant water shutoff profile control agent}

High temperature resistant inorganic gel plugging agent.High temperature resistant inorganic gel plugging agent was developed for heavy oil stimulation water plugging, of good temperature resistance, suitable for steam injection. The plugging agent is composed of binder, activator, dispersing agent, stabilizer and so on, is gray solid powder, is used with the stability suspension liquid of $10 \%-20 \%$. The main components of binder is low carbon iron-rich sulfoaluminate, has potential hardening properties, chemical inertness under normal temperature and pressure. Temperature over $40^{\circ} \mathrm{C}$ and special inorganic/organic composite excitation activator are two necessary conditions to inspire the reactivity. Under formation condition, water plugging agent passes through the activation phase, the hard period, strength growth stage, generates high-strength gel, which lowers the permeability of water production interval to achieve water plugging[3].

High temperature resistant inorganic gel plugging agent gelation test. Gelation time of plugging agent depends on temperature and activator concentration, two factors. Gelation temperature is related to reservoir depth. Set experiment temperature to $50^{\circ} \mathrm{C}$, changed the activator concentration to determinate plugging agent gel time, gelation standard took plugging agent losing liquidity as the 
initial setting time, completely solidified as final setting time. The results are shown in Figure 1.

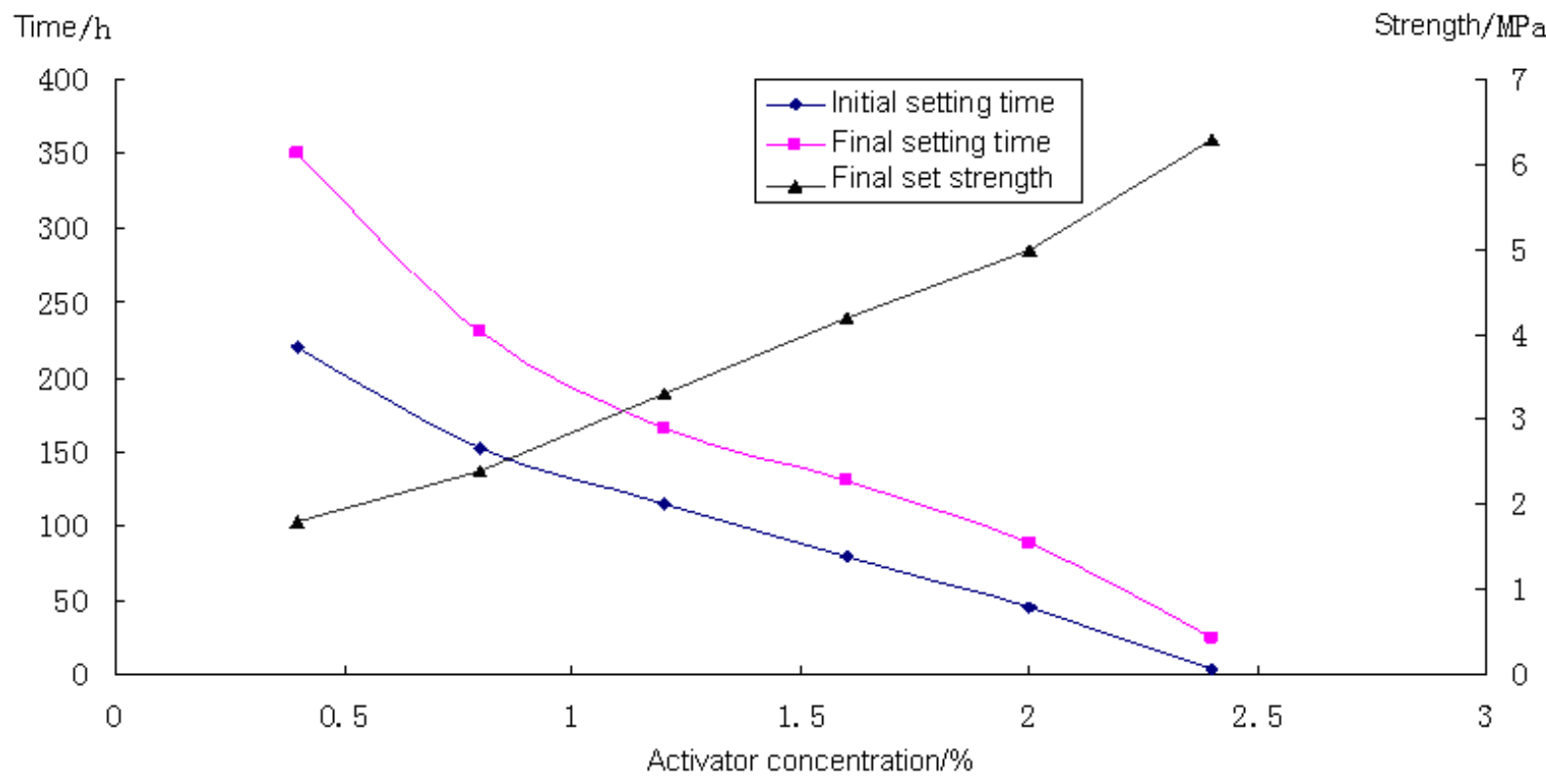

Fig.1. Activator concentration and the gel time and gel strength curve

From figure 1 , it showed that when the activator concentration was less than $0.5 \%$, gel time of plugging agent was a long time and a low gel strength, When the activator concentration was above $2.5 \%$, gel speed was too fast, lost application value. Therefore, the use of the activator concentration should be adjusted according to the requirements of injection time between $0.5 \%-2.5 \%$.

High temperature resistant inorganic gel plugging agent high temperature block off test. Under $50^{\circ} \mathrm{C}$, squeezed high temperature resistant inorganic gel plugging agent into the artificial cores of different permeability, closed import and export gate, kept constant temperature for $96 \mathrm{~h}$ to completely gel.Then put rock core in the autoclave at $350^{\circ} \mathrm{C}$, kept constant temperature for 7 days, took out and measured its permeability, calculated rock core plugging ratio, the results are shown in Table 1.

Tab.1. Plugging performance measurement of high temperature resistant inorganic gel plugging agent

\begin{tabular}{|c|c|c|c|c|}
\hline \multirow{2}{*}{$\begin{array}{l}\mathrm{C} \\
\text { ore } \\
\text { No. }\end{array}$} & \multicolumn{3}{|c|}{ The water phase permeability of core $\left(\mathrm{um}^{2}\right)$} & \multirow[b]{2}{*}{$\begin{array}{l}\text { High temperature } \\
\text { plugging rate }(\%)\end{array}$} \\
\hline & $\begin{array}{r}\text { Before } \\
\text { plugging }\end{array}$ & $\begin{array}{c}\text { After } \\
\text { plugging }\left(50^{\circ} \mathrm{C}\right)\end{array}$ & $\begin{array}{c}\text { Constant } \\
\text { temperature } 350^{\circ} \mathrm{C}\end{array}$ & \\
\hline 1 & 0.297 & 0.011 & 0.019 & 93.6 \\
\hline 2 & 0.863 & 0.015 & 0.021 & 97.6 \\
\hline 3 & 1.404 & 0.012 & 0.033 & 97.6 \\
\hline 4 & 2.130 & 0.018 & 0.028 & 98.7 \\
\hline
\end{tabular}

From the experimental results, not only high permeability layer but also middle and low permeability formation, after plugging agent treatment, can achieve high plugging ratio, and are suitable for high temperature steam injection conditions. In addition, the salt tolerance of plugging agent, compatibility and other performances were summarized as follows:1) Gel time: 8 240 h is adjustable(2) Gel temperature range: $40 \sim 80{ }^{\circ} \mathrm{C}(3)$ Plugging ratio: $>90 \%$ (4) Breakthrough pressure: $8 \mathrm{mpa} / \mathrm{m}(5)$ Temperature resistance: $350^{\circ} \mathrm{C}$. 


\section{High temperature temporary plugging agent}

According to the design requirements, the temporary plugging agent can lose plugging ability in different time, can make the high permeable zone regain its permeability to realize inject respectively and joint exploitation[4]. High temperature temporary plugging agent is make up of modified resin, cross-linking agent and solid phase particles.Modified resin in the presence of crosslinking agent can gel under $25^{\circ} \mathrm{C}$, gel time is for $6 \sim 16$ hours adjustable, Its core breakthrough pressure is greater than $10 \mathrm{MPa} / \mathrm{m}$, can block off high permeable zone effectively. Under the condition of steam injection, Plugging ratio of high temperature temporary plugging agent remained $93.0 \%$ after $240 \mathrm{~h}$, plugging ratio fell to $43.0 \%$ after $480 \mathrm{~h}$.

The relationship experiment between heating time and plugging ratio of high temperature temporary plugging agent is as follows:

1.Experimental apparatus: injection water tank used for hydraulic system, precision metering pump, Steam generator, plugging agent tank;

Data acquisition system: differential pressure sensors, temperature sensors, computer, high temperature oven (room temperature to $400^{\circ} \mathrm{C}$ );

Core model: $\mathrm{L}=45 \mathrm{~cm}, \phi=3.7 \mathrm{~cm}$;

Sample collection system: electronic balance, backpressure regulator.

2.Experimental conditions: Quartz sand filling model (porosity $\phi=35 \%$, permeability $\mathrm{K}=4.8$ $\mathrm{um}^{2}$ ); Connect test process, pressure test requirement is $16.5 \mathrm{MPa}$ and don't leak, the intensity of steam injection boiler emissions is $20 \mathrm{t} / \mathrm{h}$, convert to steam injection intensity of model is not less than $12 \mathrm{~g} /\left(\mathrm{h} \cdot \mathrm{cm}^{2}\right)$; Reservoir parameters: oil layer perforated thickness is $50 \mathrm{~m}$, used degree is $30 \%$, porosity is $30 \%$, temporary plugging radius is $1.5 \mathrm{~m}$. Keep the temperature at $50 \sim 55^{\circ} \mathrm{C}$;

3.Experimental procedure: Injected temporary plugging agent measured to the model, coagulate for 48 hours, then injected steam of $350^{\circ} \mathrm{C}$ to model, steam intensity was $12 \mathrm{~g} /\left(\mathrm{h} \cdot \mathrm{cm}^{2}\right)$, collected core permeability at different time, calculated plugging ratio.

4.the result:

At $350^{\circ} \mathrm{C}$, the decline of block ratio has a breakdown process from $270 \mathrm{~h}$ to $480 \mathrm{~h}$, contact area between the temporary plugging agent and injected steam is expanded to a certain value, temporary plugging agent is washed and corroded, degradation process has a sharp increase, then dissolution quantity is stable.

Tab.2. Relations of steam injection time and plugging rate

\begin{tabular}{|c|r|r|r|r|r|r|r|r|r|r|}
\hline $\begin{array}{c}\text { Steam } \\
\text { injection time (h) }\end{array}$ & 0 & 24 & 72 & 144 & 192 & 240 & 288 & 312 & 360 & 480 \\
\hline $\begin{array}{c}\text { plugging rate } \\
(\%)\end{array}$ & 100 & 99.9 & 99.0 & 97.2 & 95.1 & 93.0 & 88.1 & 68.7 & 47.1 & 43.0 \\
\hline
\end{tabular}

Through the adjustment of temporary plugging agent formula, temporary plugging time is adjustable within a certain range to realize inject respectively and joint exploitation. Part of chemical agent in steam injection Wells need to have both steam temperature resistance and gradual degradation under the temperature of the steam injection, the patent technology can be applied to this situation.

\section{Field test}

High temperature resistance water shutoff profile control agent was carried out cumulative 116 Wells in Huanxiling, Shuguang, Gaosheng production plants, which effectively improved uneven steam absorption between layers oil for the high round time steam stimulation well, improved the steam swept volume and heavy oil flowback ability, solved interwell steam channeling, greatly improved steam stimulation effect of heavy oil reservoir in Liaohe oilfield. Measure effective ratio was $93 \%$, with average incremental oil production of $571.7 \mathrm{t}$ per well, oil output increased $0.7 \sim 1.8 \mathrm{t}$ in a single well per day, measure effect was ideal. 
Shu 1-37-43 well. After nine round steam injection, the well cycle production was low, production decline is very fast. It was influenced by adjacent wells steam channeling, which led water rise rapidly, output declined. Composite high temperature profile control technology was used before the 10th round of steam injection, profile control was principal, water shutoff was subsidiary, blocked off steam channeling channel, enlarged swept volume, improved steam injection efficiency.

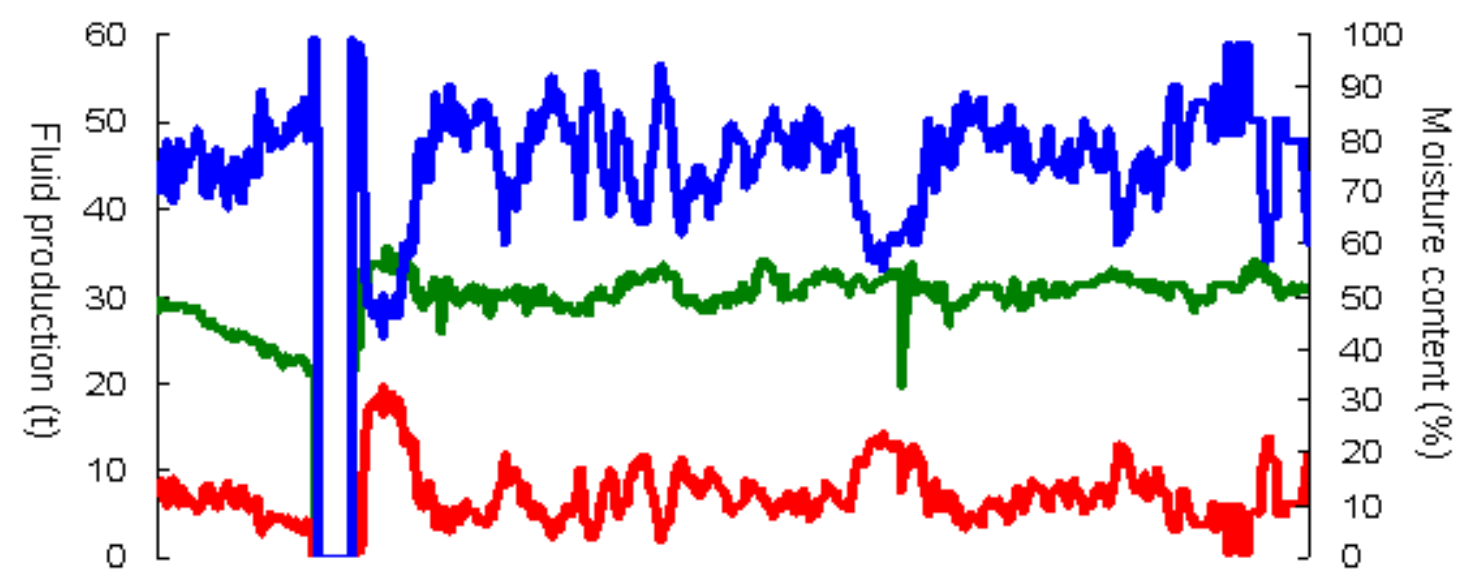

Fig.2. Production curve of Shu 1-37-43 well

Gao 3-6-231 well.After five rounds steam injection, cycle production and water recovery rate were low, steam stimulation effect was poor. Main reason was after several rounds of steam injection production, high seepage channel has been formed in high used horizon, residual oil content is low, steam injection formed invalid stimulation; vapor absorption quantity is little at unused formation, the effect is poor, residual oil content is high but can't mine effectively.Before 6 rounds steam injection, using compound high temperature profile control, inorganic gel sealing and aid mining agent, adjust the steam absorption profile, improve crude oil mobility, increase single well used degree.

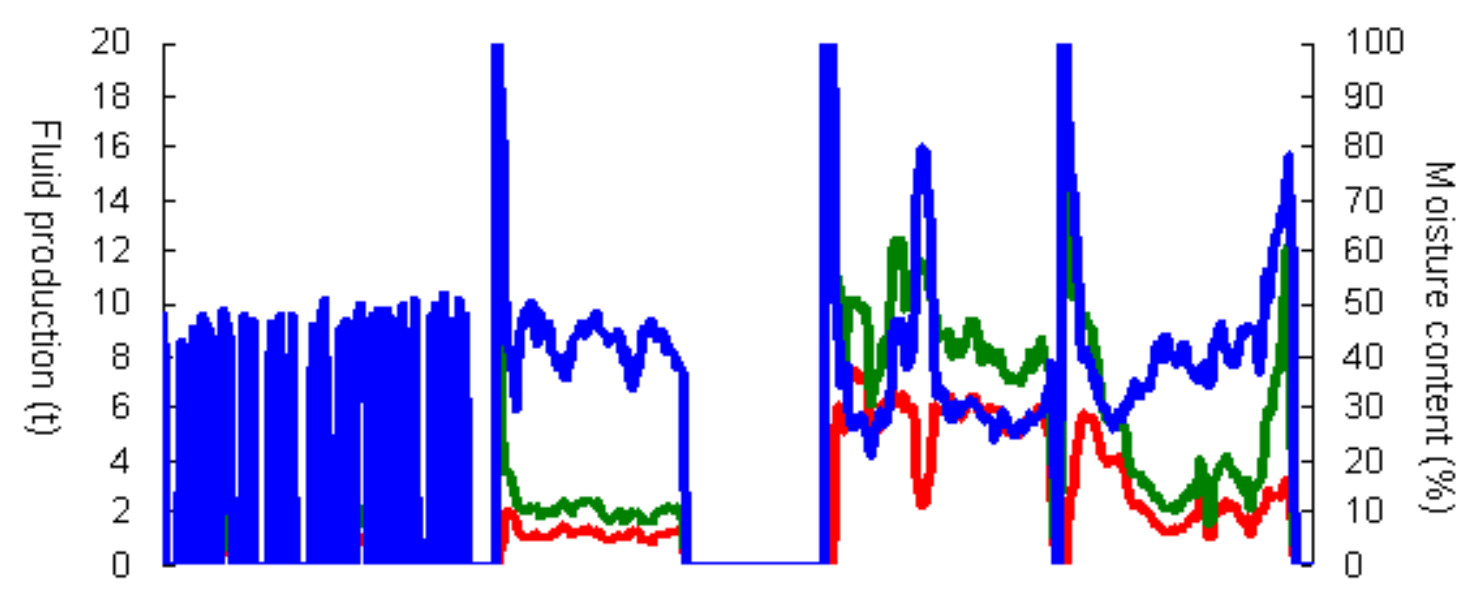

Fig.3. Production curve of Gao 3-6-231 well

\section{Conclusion}

(1) For the characteristics of Liaohe oilfield heavy oil reservoir stimulation exploitation, a series of blocking agent formula system is developed, which make the plugging agent temperature resistant up to $350^{\circ} \mathrm{C}$, has solved high temperature water shutoff profile control problem of the heavy oil steam injection wells. 
(2) According to different well conditions, using composite plugging technology, different types blocking agent are combined organically, which can meet the needs to various well conditions.

(3) High temperature profile control agent has certain resistance to steam erosion intensity. After implementation, the steam don't enter into the blocked horizon to adjust steam absorption profile and improve the effect of steam injection, to improve reservoir producing extent.

\section{References}

[1]Guynn, Herbert G Amino. Resins crosslinked polyer gels for permeability profile control.[P]. US:4834180.1989-05.

[2]BenWang, Larry W. Lake and Gary A. Pope. Development and aplliaction of a streamline micellar/polymer simulator. SPE10290.

[3]Jenny Tai Liang, Haiwaysun, SerightR S. Why do gels reduced water permeability more than oil permeability[P]. SPE:27289(Apr.1994):17-20.

[4]White J L, Goddard J E, Phillips H M. Use of polymers to control water production in oil well[J]. Journal of Petroleum Technology, 1973,35(4):143-150. 